\title{
Milliequivalent per 24 Hours
}

National Cancer Institute

\section{Source}

National Cancer Institute. Milliequivalent per 24 Hours. NCI Thesaurus. Code 667471.

A unit of relative amount of substance flow rate equivalent to the rate at which one

thousandth of an equivalent of substance crosses a given surface or is delivered to a given object or space over a period of time equal to twenty four hours. 